\title{
Enhanced Exciton-Phonon Coupling in Colloidal Type-II CdTe-CdSe Heteronanocrystals
}

\author{
Esther Groeneveld and Celso de Mello Donegá* \\ Condensed Matter and Interfaces, Debye Institute for Nanomaterials Science, Utrecht University, 3508 TA Utrecht, Netherlands
}

Supporting Information

ABSTRACT: In this paper, the observation of LO-phonon progressions in the low temperature $(4.2 \mathrm{~K})$ photoluminescence excitation spectra of different types of colloidal NCs is reported. The strength of the exciton-LO-phonon coupling at $4.2 \mathrm{~K}$, as reflected by the Huang-Rhys parameter $S$, increases from CdSe QDs $(S=0.8-1.0)$ to Type- $\mathrm{I}^{1 / 2} \mathrm{CdTe} / \mathrm{CdSe} \mathrm{HNCs}(S=1.5)$, and from these to Type-II CdTe/CdSe HNCs $(S=2.9)$. This trend is explained by the decrease in the electron-hole wave function overlap that accompanies the gradual increase in the CdSe volume fraction in the HNCs. The decrease in the electron-hole overlap increases the exciton polarization, which in turn enhances the exciton-LO-phonon coupling via the Fröhlich interaction. The results provide novel insights into the nature of spatially indirect exciton transitions and show that compositional control of semiconductor heteronanocrystals can be used as a very effective tool to tailor the exciton-phonon coupling strength of nanoscale excitons.

\section{INTRODUCTION}

Colloidal semiconductor nanocrystals (NCs) have, as a result of quantum confinement effects, size and shape dependent properties. ${ }^{1}$ Moreover, they offer the prospect of solution processing and easy control over surface functionality. Colloidal NCs consisting of two (or more) different semiconductors joined by heterointerfaces (i.e., heteronanocrystals, HNCs) offer even more possibilities regarding property engineering. ${ }^{1}$ The offsets between the energy levels of the materials that are combined at the heterointerfaces in HNCs can be tuned by controlling the composition, size, and shape of each component, allowing the carrier localization regime to be controlled. ${ }^{1}$ In Type-I HNCs the energy offsets are such that both carriers are confined in the same material (typically the core in core/shell HNCs). In contrast, the alignment between the energy levels of the different components in Type-II HNCs is staggered, leading to the formation of a spatially indirect exciton, in which the electron and hole wave functions are primarily localized in different segments of the HNC. In Type$\mathrm{I}^{1 / 2}$ HNCs one carrier is delocalized over the whole HNC, whereas the other is localized in one of the components. Control over the energy offsets in HNCs thus allows the electron-hole spatial overlap to be tailored, yielding a remarkable degree of control over the properties of nanoscale excitons. $^{1-7}$

These characteristics have turned semiconductor NCs and HNCs into promising materials for a variety of technologies, ranging from lighting and optoelectronics to biomedical imaging, photovoltaics, and thermoelectrics. ${ }^{8,9}$ A comprehensive understanding of nanoscale excitons is a crucial prerequisite for all of these potential applications. This has stimulated a vast research effort to unravel the fundamental properties of semiconductor nanostructures, leading to extensive investigations and great advances in the last few decades. ${ }^{1,3,8-13}$ Phonons have a pervasive role in semiconductor materials, having an impact on a wide variety of properties, such as charge carrier mobility, heat transport, exciton relaxation and lifetimes, and superconductivity. ${ }^{14}$ The interaction between phonons and electrons (or excitons) in nanoscale semiconductors is expected to differ from that in bulk materials due to both quantum confinement effects on the excitonic energy levels and dimensional confinement of phonon modes. ${ }^{14}$ The electron-phonon (el-ph) coupling is thus vital to our understanding of the properties of nanoscale systems. Coupling of the electron and/or hole to phonons provides an important energy relaxation pathway, being thus essential to a number of photophysical processes in semiconductor nanostructures (viz., exciton and multiexciton relaxation dynamics, hot carrier cooling, carrier multiplication, interfacial charge separation, etc.). 2,3,10,12,13,15-18 el-ph coupling is also crucial for thermal transport processes ${ }^{19}$ and underpins the temperature dependence of both electron transport and nonradiative energy transfer processes in colloidal quantum dot (QD) superlattices. $^{20-22}$ Moreover, coupling to acoustic phonon modes determines the homogeneous linewidths of optical transitions and contributes to the resonant Stokes shift, ${ }^{23}$ whereas coupling to longitudinal optical (LO) phonon modes has been observed to relax selection rules, yielding momentum-conserving optical transitions (i.e., phonon-assisted transitions or phonon replicas) from the so-called "dark exciton states" in semiconductor QDs and quantum rods. ${ }^{24-28}$ Interestingly, it has been recently proposed that coupling to low energy acoustic phonons

Received: April 4, 2012

Revised: July 2, 2012

Published: July 4, 2012 
transiently mixes "dark" and "bright" exciton states, ${ }^{29,30}$ thereby increasing the radiative decay rates of QDs and leading to a pronounced temperature dependence of the exciton lifetimes below $20 \mathrm{~K}^{30}$

The critical impact of the el-ph coupling on the properties of nanoscale excitons has spurred a large number of theoreti$\mathrm{cal}^{31-37}$ and experimental ${ }^{12,13,24,25,32,37-55}$ studies on the el-ph and the exciton-phonon (ex-ph) coupling strength in semiconductor NCs of a variety of materials, such as $\mathrm{Si}^{38}$ $\mathrm{Ge}^{39} \mathrm{CdS},{ }^{40} \mathrm{InP},{ }^{41} \mathrm{PbS},{ }^{42} \mathrm{ZnTe}^{54} \mathrm{CdSe},{ }^{12,13,24,25,32,43-49}$ $\mathrm{CdSe} / \mathrm{ZnS},{ }^{28,44} \mathrm{CdSe} /(\mathrm{Cd}, \mathrm{Zn}) \mathrm{S},{ }^{27,50,55} \mathrm{InAs} / \mathrm{GaAs},{ }^{37,51,52}$ and (Ga,In)N/GaN. ${ }^{53}$ To date, however, only single composition NCs and Type-I heterostructures have been addressed. A study of the ex-ph coupling in Type-II HNCs is still lacking, despite the importance of these materials from both fundamental and applied viewpoints. ${ }^{1-6}$ In this work, we address this need by investigating phonon-assisted transitions (LO-phonon replicas) in the low temperature $(4.2 \mathrm{~K})$ photoluminescence excitation (PLE) spectra of colloidal Type- $\mathrm{I}^{1 / 2}$ and Type-II CdTe/CdSe HNCs. The relative intensities of the LO-phonon replicas are used to derive the Huang-Rhys parameter $S$, which reflects the strength of the ex-ph coupling. Colloidal CdTe/CdSe HNCs are chosen for this study for a number of reasons. First, highly luminescent colloidal CdTe/CdSe HNCs have become recently available $^{56}$ and were thoroughly investigated by our group as a model system to unravel the formation of spatially indirect nanoscale excitons as a function of the size, shape, and composition of the HNC. ${ }^{57}$ Second, CdTe and CdSe QDs are model systems themselves, being the most investigated and best understood colloidal QDs. In particular, the el-ph and ex-ph coupling strengths of CdSe NCs have been extensively investigated over the last two decades by a variety of experimental techniques, ${ }^{12,13,24,25,32,43-49}$ including PLE and fluorescence line narrowing (FLN). ${ }^{24-27}$ Colloidal CdSe QDs are therefore also investigated in this work as a reference for the ex-ph coupling strength. Our results clearly show that the ex-ph coupling strength increases with increasing spatial separation of the electron and hole wave functions.

\section{EXPERIMENTAL METHODS}

2.1. Sample Preparation. In this work colloidal suspensions of organically capped CdSe QDs and CdTe/ $\mathrm{CdSe}$ HNCs were investigated. Two different samples of CdSe QDs were used: $3 \mathrm{~nm}( \pm 10 \%)$ diameter spherical NCs capped with oleic acid $(\mathrm{OA})$ and $4.3 \mathrm{~nm}( \pm 5 \%)$ diameter prolate (aspect ratio of 1.2) NCs capped with trioctylphosphineoxide (TOPO) and hexadecylamine (HDA). TOPO-HDA capped $\mathrm{CdSe}$ QDs were synthesized as reported previously. ${ }^{58} \mathrm{OA}$ capped CdSe QDs were synthesized via an adaptation of the method reported by $\mathrm{Qu}$ and Peng. ${ }^{59}$ Details of this adapted method are given in the Supporting Information. The colloidal $\mathrm{CdTe} / \mathrm{CdSe} \mathrm{HNCs}$ are capped by TOPO-HDA and were prepared by a multistage seeded growth approach described in detail previously. ${ }^{56}$ Two different CdTe/CdSe HNC samples were used, both grown from the same batch of $2.6 \mathrm{~nm}$ diameter CdTe QD seeds: prolate Type- $\mathrm{I}^{1 / 2}$ HNCs (4.4 nm long, 39\% CdSe volume fraction, here on referred to as Type- $\mathrm{I}^{1 / 2} \mathrm{CdTe} /$ CdSe HNC) and bipod shaped Type-II HNCs (pod length: 8 $\mathrm{nm}$; pod diameter: $2.9 \mathrm{~nm}$; $88 \%$ CdSe volume fraction, here on referred to as Type-II CdTe/CdSe HNC; see the Supporting Information, Table S-1, for further details). These two samples have been thoroughly investigated in previous works by our group. $^{56,57}$
2.2. Optical Spectroscopy. Photoluminescence (PL) and PL excitation (PLE) spectra were acquired using an Edinburgh Instruments FLS920 Spectrofluorimeter equipped with a 450 $\mathrm{W}$ xenon lamp as the excitation source and double grating monochromators. The spectral resolution was $1 \mathrm{~nm}(2-4 \mathrm{meV}$, depending on the spectral region) for the excitation monochromator and $0.3-0.5 \mathrm{~nm}(0.6-2 \mathrm{meV}$, depending on the spectral region) for the emission monochromator. The excitation power was $100-200 \mathrm{~mW} / \mathrm{cm}^{2}$. The spectra were corrected for the instrumental response and the spectral dependence of the excitation lamp intensity. Samples for optical measurements were prepared by directly dissolving the crude reaction mixture in anhydrous toluene in a glovebox under nitrogen $\left(<1 \mathrm{ppm} \mathrm{H}_{2} \mathrm{O},<5 \mathrm{ppm} \mathrm{O}_{2}\right)$. In order to avoid energy transfer between the NCs and deviations from linearity in the PLE spectra, all optical measurements were carried out on samples with a low optical density $(\leq 0.05$ at the lowest energy absorption maximum). For low temperature measurements the sample was contained in a sealed quartz cuvette (optical path of $2 \mathrm{~mm}$ ) and mounted in a continuous He-flow cryostat allowing for measurements down to $4.2 \mathrm{~K}$.

\section{RESULTS AND DISCUSSION}

3.1. LO-Phonon Replicas in the Low Temperature Optical Spectra of Colloidal NCs. Figure 1 presents the low temperature $(4.2 \mathrm{~K}) \mathrm{PL}$ and PLE spectra of the CdSe QDs and $\mathrm{CdTe} / \mathrm{CdSe}$ HNCs investigated in this work. The PL spectra consist of a single peak, whereas the PLE spectra comprise a number of different absorption transitions. An assignment for the transitions observed in the spectra will be presented later in this paper (sections 3.2 and 3.3 below). It is interesting to note that fine-structure is clearly observed near the low energy edge of the PLE spectra (see Figure 2 for a detailed view), in contrast to the featureless PL spectra. The lack of fine-structure in the PL spectra shown in Figure 1 is due to the fact that the samples were excited far above the band gap (viz., $440 \mathrm{~nm}$ ). Therefore, the spectra reflect the full size and shape distribution of the NC (HNC) ensemble. It should be noted that the ensemble PL spectrum is however red-shifted from the statistically weighted maximum, since the density of states far above the band-edges is proportional to the volume of the NC (or HNC), ${ }^{60}$ and therefore, larger NCs in the ensemble have relatively larger contributions to the full PL spectrum.

PLE spectroscopy is ideally suited to reveal the near bandedge fine-structure, since it effectively narrows the dispersion of the NCs (or HNCs) probed. This is done by monitoring a narrow spectral band of the full ensemble PL, while scanning the excitation energy with high resolution. In this way, subensembles of NCs (HNCs) are spectrally selected, thereby minimizing the impact of size, shape, and composition inhomogeneities on the transitions observed. The use of samples with low optical densities further minimizes the effects of polydispersity, since it prevents energy transfer from smaller to larger NCs in the ensemble. Another useful feature of the PLE technique is that only emitting NCs (HNCs) contribute to the spectra, ensuring that only information over the most efficient NCs (HNCs) in the ensemble is collected. Moreover, $\mathrm{PL}$ associated with radiative recombination in defects or surface states (i.e., "trap PL") occurs at lower energies than the bandedge PL and can thus be discarded by spectral selection. PLE spectroscopy can therefore effectively exclude defective NCs (HNCs) from the optical sampling process. However, only at low temperatures the benefits of PLE spectroscopy can be fully 

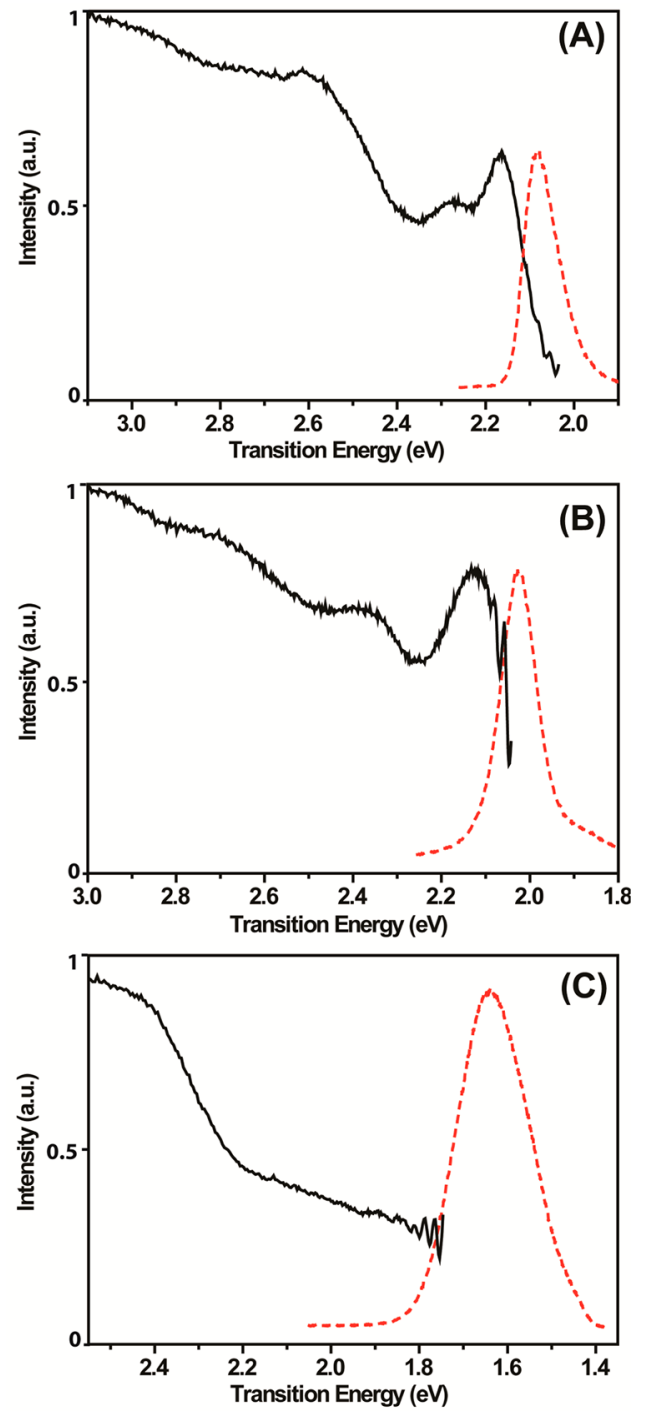

Figure 1. Low temperature $(4.2 \mathrm{~K})$ PL (dashed red lines, $\lambda_{\text {exc }}=440$ $\mathrm{nm}$ ) and PLE (solid black lines) spectra of (A) $4.3 \mathrm{~nm}$ diameter CdSe NCs $\left(\lambda_{\text {em }}=614 \mathrm{~nm}\right),($ B $)$ CdTe $/$ CdSe Type-I ${ }^{1 / 2}$ HNCs $\left(\lambda_{\text {em }}=612\right.$ $\mathrm{nm})$, and (C) CdTe/CdSe Type-II HNCs $\left(\lambda_{\mathrm{em}}=715 \mathrm{~nm}\right)$.

harvested. The bandwidth of exciton transitions in PL and PLE spectra is determined by homogeneous and inhomogeneous broadening. Inhomogeneous broadening is caused by size, shape, and composition inhomogeneities within the ensemble, whereas homogeneous broadening is due to intrinsic processes, such as exciton dephasing by coupling to acoustic phonons. ${ }^{23}$ The homogeneous linewidths thus decrease with decreasing temperatures as a result of the lower phonon populations, ${ }^{23}$ reaching values as narrow as $10-200 \mu \mathrm{eV}$ for single CdSe QDs at $2 \mathrm{~K}^{28,55}$ Therefore, at sufficiently low temperatures, the linewidths of ensembles of QDs are dominated by the inhomogeneous broadening contribution, allowing one to use PLE spectroscopy to select narrow subensembles of emitting NCs (HNCs), effectively minimizing the impact of polydispersity and allowing the fine-structure of the spectral transitions to be observed.

As can be seen in Figure 1, the PLE spectra consist of several partially overlapping broad bands and a number of weaker and narrow lines near the low energy edge. As the monitored PL is moved to lower energies the broad features broaden and
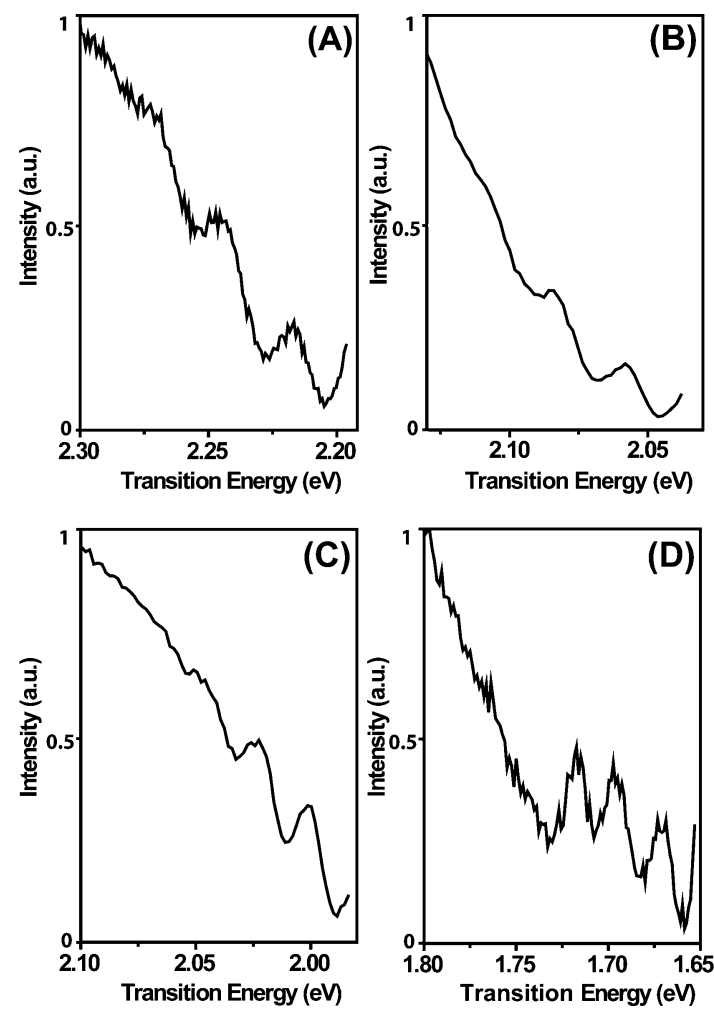

Figure 2. Low energy edge of the low temperature (4.2 K) PLE spectra of (A) $3 \mathrm{~nm}$ diameter CdSe NCs $\left(\lambda_{\mathrm{em}}=568 \mathrm{~nm}\right)$, (B) $4.3 \mathrm{~nm}$ diameter CdSe NCs $\left(\lambda_{\mathrm{em}}=614 \mathrm{~nm}\right),(\mathrm{C}) \mathrm{CdTe} / \mathrm{CdSe}$ Type-I $\mathrm{I}^{1 / 2}$ HNCs $\left(\lambda_{\mathrm{em}}=630 \mathrm{~nm}\right)$, and (D) CdTe/CdSe Type-II HNCs $\left(\lambda_{\mathrm{em}}=\right.$ $756 \mathrm{~nm})$.

become relatively weaker, whereas the narrow fine-structure features near the low energy edge of the PLE spectra become more pronounced (Supporting Information, Figure S1). This is due to a combination of effects (see the Supporting Information, Figure S1, for details). First, when the PL is monitored on the blue edge of the full ensemble PL, the smallest NCs in the ensemble are probed, whereas the largest NCs are probed when monitoring the red edge of the full ensemble PL (Figure S1). Because larger NCs (smaller energy gaps) can reabsorb the emission of smaller NCs (larger energy gaps), the PLE spectra of the largest NCs in the ensemble will consist of a convolution of absorption transitions leading to direct excitation of the NCs (i.e., the true PLE spectrum of the largest NCs) and absorption transitions of smaller NCs that indirectly excite the largest NCs via emission reabsorption. Therefore, the features in the PLE spectrum of the largest NCs become broader, particularly at higher energies, where the spectral overlap with the smaller NCs is more pronounced. For this reason, the highest spectral resolution in PLE for the strong and broad features is obtained while probing the smallest NCs in the ensemble (i.e., the blue edge of the full ensemble PL), because they are not affected by reabsorption effects. However, the near band-edge fine-structure is best observed while monitoring the largest NCs in the ensemble (i.e., the red edge of the full ensemble PL), because they have the smallest band-gaps. Therefore, their near band-edge transitions are not affected by spectral overlap with transitions of smaller NCs (Figure S1). In practice, the attainable spectral resolution is limited by a number of constraints, and therefore, some spectral overlap still occurs. 
Moreover, saturation effects in the PLE spectra also play a role. These effects originate from the fact that at sufficiently high optical densities the relationship between the absorption cross section and the concentration of the absorber becomes nonlinear, with larger deviations for stronger transitions. ${ }^{61}$ Therefore, the relative intensities of different transitions in a PLE spectrum become distorted. This effect is important in the low temperature PLE spectra of colloidal suspensions of NCs, because the solution freezes out as a polycrystalline material. Light scattering at the intergrain boundaries leads to effective optical path lengths significantly longer than the thickness of the cell containing the sample. The effective optical path length of photons capable of exciting stronger transitions will then be shorter than that of weakly absorbed photons (Supporting Information, Figure S1). As a result, the relative intensities in the PLE spectra are distorted, with weaker transitions appearing relatively stronger (see comparison between PLE spectra at low temperatures and at room temperature, where light scattering effects are absent, Supporting Information, Figure S1). This effect also favors the observation of the near band-edge finestructure in the PLE spectrum of the largest NCs in the ensemble, since it enhances their relative intensity with respect to the broad background formed by the higher energy (and stronger) transitions, which, as explained above, contain contributions from all the emitting NCs in the ensemble that are smaller than the ones being monitored.

The low energy edge of the PLE spectra of the studied samples is shown in more detail in Figure 2. Despite the pronounced differences between the overall features in the PLE spectra of CdSe QDs (Figure 1A), Type-I ${ }^{1 / 2} \mathrm{CdTe} / \mathrm{CdSe}$ HNCs (Figure 1B), and Type-II CdTe/CdSe HNCs (Figure 1C), the fine-structure near the lowest energy absorption edge is strikingly similar in all four cases (Figure 2), consisting of a series of equally spaced narrow lines. The spacing between the lines (viz., $26 \pm 1 \mathrm{meV}$ for CdSe QDs, Figure $2 \mathrm{~A}, \mathrm{~B} ; 23 \pm 1$ $\mathrm{meV}$ for $\mathrm{CdTe} / \mathrm{CdSe}$ Type- $\mathrm{I}^{1 / 2} \mathrm{HNCs}$, Figure $2 \mathrm{C}$, and $24 \pm 1$ $\mathrm{meV}$ for CdTe/CdSe Type-II HNCs, Figure 2D) corresponds well to the frequency of longitudinal optical (LO) phonon modes in bulk CdSe $(26 \mathrm{meV}){ }^{62}$ especially for CdSe QDs. The slightly smaller spacing observed in the spectra of the CdTe/ CdSe HNCs is in fact very close to the volume average between the LO-phonon frequencies of bulk CdTe $(21 \mathrm{meV})^{63}$ and bulk CdSe, which corresponds to $23 \mathrm{meV}$ for CdTe/CdSe Type- $\mathrm{I}^{1 / 2}$ HNCs (39\% CdSe, 61\% CdTe) and to $25 \mathrm{meV}$ for CdTe/ CdSe Type-II HNCs (88\% CdSe, 22\% CdTe).

We note that fine-structure can also be observed in the low temperature PL spectra upon selective excitation of narrow subensembles of NCs. This is typically achieved by using spectrally narrow lasers as the excitation source, yielding the socalled fluorescence line narrowed (FLN) spectra, ${ }^{24-27}$ but can also be (partially) observed by using narrow excitation and emission slits and near band-edge excitation (see, e.g., the Supporting Information, Figure S2). The spacing between the fine-structure features observed in the low temperature PL spectra (Supporting Information, Figure S2) is the same as that observed in the PLE spectra. The near band-edge fine-structure observed in both the PLE and PL spectra can thus be assigned to a LO-phonon progression. In the present work, we will focus on the PLE spectra because they are better suited to quantitatively investigate the near band-edge fine-structure, for reasons to be made clear below.

The main difference between the LO-phonon progressions observed for the different types of NCs is the relative intensities of the phonon replicas, which implies different excitonphonon coupling strengths. Another difference is the relative contribution of the spectrally broad feature onto which the LOphonon progression is superposed. To understand these differences and to allow a proper quantitative analysis of the LO-phonon replicas, it is necessary to first clearly establish the nature of the emitting state probed by the PLE spectra and of the exciton absorption transitions that populate it. The near band-edge states of the fully relaxed exciton are of particular interest, since the PLE spectra probed the near band edge PL and the measurements were carried out at $4.2 \mathrm{~K}$, under low power $\mathrm{CW}$ excitation, and using time integrated spectral acquisition. This analysis will be carried out in the next two sections (3.2 and 3.3). The intensity of the LO-phonon replicas will be addressed in section 3.4.

3.2. Exciton Transitions and Exciton Relaxation in Colloidal CdSe QDs. We start with the CdSe QDs, since they have been thoroughly investigated over the last few decades and are therefore well understood. The absorption transitions observed for the CdSe QDs (Figure 1) can be assigned based on previous experimental and theoretical work: ${ }^{64} 1 \mathrm{~S}_{3 / 2(\mathrm{~h})} \rightarrow$ $1 \mathrm{~S}_{(\mathrm{e})}(2.16 \mathrm{eV}), 2 \mathrm{~S}_{3 / 2(\mathrm{~h})} \rightarrow 1 \mathrm{~S}_{(\mathrm{e})}(2.28 \mathrm{eV})$, and $1 \mathrm{P}_{3 / 2(\mathrm{~h}) ; 1 / 2(\mathrm{~h})} \rightarrow$ $1 \mathrm{P}_{(\mathrm{e})}(2.6 \mathrm{eV})$. The emission transition is $1 S_{(\mathrm{e})} \rightarrow 1 S_{3 / 2(\mathrm{~h})}$. It can thus be concluded that the LO-phonon progression observed in Figures 1 and 2 is associated with the $1 S_{3 / 2(\mathrm{~h})} \rightarrow$ $1 \mathrm{~S}_{(\mathrm{e})}$ transition. The lowest electron state $\left(1 \mathrm{~S}_{(\mathrm{e})}\right)$ is doubly degenerate with respect to its spin projection, whereas the lowest hole state $\left(1 S_{3 / 2(h)}\right)$ is 4 -fold degenerate with respect to the projection of its total angular momentum. The $1 S_{(\mathrm{e})} 1 S_{3 / 2(\mathrm{~h})}$ exciton state of spherical CdSe (and CdTe) NCs with cubic structure (i.e., zinc blend) is therefore 8 -fold degenerate. ${ }^{64,65}$ In the framework of the effective mass approximation (EMA) this degeneracy is partially lifted in wurtzite CdSe QDs, due to the combined effects of crystal field asymmetry, shape anisotropy, and electron-hole exchange interaction, yielding five levels (viz., $\mathrm{E}_{0}^{\mathrm{U}}, \mathrm{E}_{+1}^{\mathrm{U}}, \mathrm{E}_{0}^{\mathrm{L}}, \mathrm{E}_{+1}^{\mathrm{L}}$, and $\mathrm{E}_{ \pm 2}$, in order of decreasing energy). ${ }^{65}$ The levels are labeled according to their total angular momentum projection along the wurtzite hexagonal axis. ${ }^{65}$ Similar results have been obtained by many-body pseudopotential $^{66}$ and semiempirical tight-binding calculations. ${ }^{67}$ The energy separation between the $1 S_{(\mathrm{e})} 1 S_{3 / 2(\mathrm{~h})}$ exciton fine structure states is observed to be strongly size and shape dependent, regardless of the theoretical framework used for the calculations. $^{65-67}$

The $1 S_{3 / 2(\mathrm{~h})} \rightarrow 1 S_{(\mathrm{e})}$ emission transition takes place from the lowest two fine-structure states $\left(\mathrm{E}_{ \pm 1}^{\mathrm{L}}\right.$ and $\left.\mathrm{E}_{ \pm 2}\right)$, typically referred to as the "bright" and "dark" excitons, since the $\mathrm{E}_{+2}$ state is optically passive in the electric dipole approximation. ${ }^{65}$ At low temperatures (below $10 \mathrm{~K}$ ) the population of the "bright exciton" ( $E_{ \pm 1}^{\mathrm{L}}$ state) is negligible, and emission will thus take place primarily from the "dark exciton" $\left(\mathrm{E}_{ \pm 2}\right.$ state), leading to long exciton lifetimes (viz., $\sim 0.2$ to $1.4 \mu \mathrm{s}$, depending on the QD size). ${ }^{30,68,69}$ Within the EMA framework the $\mathrm{E}_{ \pm 2}$ lifetime should be size independent and infinite. ${ }^{65}$ However, semiempirical tight-binding calculations have successfully reconciled experiment and theory by showing that triplet ("dark exciton") radiative lifetimes in the microsecond range can be obtained for CdSe QDs by incorporating interactions with higher exciton states and explicitly taking into account spin-orbit interactions, which lead to mixing of singlet character into nominally triplet states (i.e., the $\mathrm{E}_{ \pm 2}$ state or "dark exciton"). ${ }^{67}$

It is interesting to note that the observation of long exciton lifetimes for CdSe QDs at low temperatures was initially 
ascribed to surface localization of the photogenerated hole. ${ }^{24}$ However, subsequent work by the same group dismissed the surface-based interpretation for being inconsistent with data obtained by transient differential absorption and gated FLN spectra. $^{70}$ Since then a large body of experimental and theoretical work has established that the energetics and dynamics of the low temperature exciton PL of colloidal CdSe QDs can be understood in terms of the intrinsic bandedge exciton-fine structure. This model has successfully explained a wide variety of empirical data: the size dependence of the fine-structure observed in excitation and emission, ${ }^{25,26,65}$ the size-, temperature-, and magnetic field-dependence of the exciton lifetimes, ${ }^{19,26-28,30,65,68-71}$ and the electron and hole spin relaxation dynamics. ${ }^{72}$ Moreover, spectroscopic investigations led by a number of groups on colloidal CdSe QDs with a variety of different coatings (both organic ligands, such as TOPO, TOPO-HDA, 4-picoline, etc., and inorganic shells of $\mathrm{ZnS}$ or $\mathrm{ZnSe})$ has shown that the low temperature $(<50 \mathrm{~K})$ absorption, band-edge PL, FLN spectra, and exciton lifetimes are remarkably insensitive to the properties of the $\mathrm{QD}$ surface. ${ }^{69,73}$ These results strongly argue against the involvement of surface states, and provide compelling evidence that the exciton PL decay in colloidal CdSe QDs at low temperatures $(<50 \mathrm{~K})$ is due to an intrinsic mechanism (i.e., radiative recombination from the $\mathrm{E}_{ \pm 1}^{\mathrm{L}}$ and $\mathrm{E}_{ \pm 2}$ fine structure states).

Nevertheless, the surface has an important impact on the PL properties of colloidal QDs, since carrier trapping by surface states, surface defects, and capping ligands gives rise to nonradiative relaxation pathways, leading to PL quenching. ${ }^{1,69,70,73-78}$ Exciton trapping may also lead to defect assisted radiative recombination (the so-called trap PL). ${ }^{1}$ Trap PL in $\mathrm{CdSe}$ and CdTe QDs is characterized by a broad band emission $(\sim 300 \mathrm{meV})$ that is strongly red-shifted $(300-600 \mathrm{meV})$ with respect to the lowest energy absorption transition. ${ }^{12,58,79}$ In contrast, the band-edge PL (i.e., the $1 S_{3 / 2(\mathrm{~h})} \rightarrow 1 S_{(\mathrm{e})}$ transition) is narrow (viz., $\sim 100 \mathrm{meV}$ at $300 \mathrm{~K}$ for an ensemble with $10 \%$ polydispersity) $)^{12,57,58,69}$ and presents a very small nonresonant Stokes shift $(\sim 20 \mathrm{meV}) .{ }^{57}$ Carrier trapping and PL quenching produce very clear signatures in PL decay curves, leading to deviations from a single exponential behavior. ${ }^{69,74}$ We note that the CdSe QD samples investigated in this work have high room temperature PL QYs (>30\%), and no trap related PL, even at $4.2 \mathrm{~K}$. Moreover, the exciton lifetimes observed at $4.2 \mathrm{~K}$ are purely radiative (single exponential decays) and are consistent with values previously reported for similarly sized CdSe QDs. ${ }^{68,69,80}$

Considering the discussion above, it is clear that the emission monitored while scanning the PLE spectra of CdSe QDs at 4.2 $\mathrm{K}$ originates primarily from the $\mathrm{E}_{ \pm 2}$ fine-structure state of the $1 S_{(\mathrm{e})} 1 S_{3 / 2(\mathrm{~h})}$ exciton and that the involvement of surface states can be confidently excluded. The $1 S_{3 / 2(\mathrm{~h})} \rightarrow 1 S_{(\mathrm{e})}$ peak in the PLE spectra of CdSe QDs will thus consist of transitions involving the upper four fine-structure states (purely electronic and phonon assisted) and LO-phonon assisted $E_{ \pm 2}$ transitions. Most of the oscillator strength of the $1 S_{3 / 2(\mathrm{~h})} \rightarrow 1 \mathrm{~S}_{(\mathrm{e})}$ absorption transition is carried by the upper two fine-structure states. $^{64,65}$ The broad feature observed at $\sim 2.16 \mathrm{eV}$ in the PLE spectrum of the $4.3 \mathrm{~nm}$ diameter CdSe QDs (Figure 1A) can thus be assigned to a combination of the $E_{0}^{U}$ and $E_{ \pm 1}^{U}$ fine structure states. Possible reasons for the broad bandwidth of this spectral feature will be discussed below. The $\mathrm{E}_{0}^{\mathrm{L}}$ fine structure level is not observed, as this state is optically passive.
The energy difference between the $\mathrm{E}_{ \pm 1}^{\mathrm{L}}$ level and the $\mathrm{E}_{ \pm 2}$ finestructure states (the so-called "dark-bright" splitting) is very small and strongly size-dependent. ${ }^{64,65,69}$ The "dark-bright" splitting is equivalent to the resonant Stokes shift observed between the excitation and the zero-phonon line frequencies in FLN spectra and has been reported to increase from 3 to 8 $\mathrm{meV}$ for CdSe QDs in the 4.5-3 nm diameter range. ${ }^{73}$ Given that the PLE spectra reported here were collected only up to $\sim 20 \mathrm{meV}$ above the monitored PL energy, we can conclude that the $\mathrm{E}_{ \pm 1}^{\mathrm{L}}$ zero-phonon line cannot be present in the PLE spectra shown in Figures 1 and 2. The narrow features near the lowest energy edge of the PLE spectra of the CdSe QD samples (Figure 2A,B) can thus be unambiguously ascribed to a LOphonon progression involving the $\mathrm{E}_{ \pm 1}^{\mathrm{L}}$ and $\mathrm{E}_{ \pm 2}$ fine-structure states, with the lowest energy line corresponding to a onephonon replica, rather than a zero-phonon line. Since the linewidths of the observed LO-phonon replicas (viz., 15-20 $\mathrm{meV}$ ) are larger than the "dark-bright" splitting, the differences between the LO-phonon progressions originating from the two states cannot be resolved.

The $\mathrm{E}_{0}^{\mathrm{U}}$ and $\mathrm{E}_{ \pm 1}^{\mathrm{U}}$ fine-structure states are not resolved in the PLE spectra due to a combination of inhomogeneous and homogeneous broadening. The larger contribution of inhomogeneous broadening is due to the fact that the spectral size selection offered by PLE spectroscopy is less effective for higher energy transitions, since the higher energy transitions of larger CdSe QDs spectrally coincide with lower energy transitions of smaller CdSe QDs (Supporting Information, Figure S1). Moreover, the homogeneous line width of the $E_{0}^{U}$ and $E_{ \pm 1}^{U}$ fine-structure states is inherently much larger than that of the $\mathrm{E}_{ \pm 1}^{\mathrm{L}}$ and $\mathrm{E}_{ \pm 2}$ levels due to lifetime broadening, since the $\mathrm{E}_{0}^{\mathrm{U}}$ and $\mathrm{E}_{ \pm 1}^{\mathrm{U}}$ levels experience a very rapid relaxation down to the lower fine-structure levels. The intra- and inter-band relaxation in CdSe QDs has been investigated in detail and is wellunderstood. $^{12,13,15,16,19,30,69,81}$ Relaxation from the higher exciton states to the lowest exciton state $\left(1 S_{(\mathrm{e})} 1 S_{3 / 2(\mathrm{~h})}\right)$ is very fast (viz., $150-500 \mathrm{fs}) .{ }^{12,13,15,16,81}$ State specific pumpprobe transient absorption measurements have determined that the $1 \mathrm{P}_{\mathrm{e}} \rightarrow 1 \mathrm{~S}_{\mathrm{e}}$ electron relaxation is size dependent (155 fs for $4 \mathrm{~nm}$ diameter and $250 \mathrm{fs}$ for $6.4 \mathrm{~nm}$ diameter CdSe QDs), while the hole relaxation is size independent ( $250 \mathrm{fs}$ for the $\left.2 S_{3 / 2(\mathrm{~h})} \rightarrow 1 S_{3 / 2(\mathrm{~h})}\right) .^{81}$ The electron relaxation rates are determined by Auger energy transfer to holes (electron-hole Auger coupling time: $1 \mathrm{ps}) .^{15,81}$ In contrast, hole relaxation has been proposed to take place primarily via coupling to phonons, either from the surface ligands or from the QD itself (both acoustic and LO-modes). ${ }^{81}$ The final intra-band relaxation step occurs within the five exciton fine-structure sublevels of the $1 \mathrm{~S}_{(\mathrm{e})} 1 \mathrm{~S}_{3 / 2(\mathrm{~h})} 16$ and is essentially determined by the hole relaxation rate. ${ }^{15,72}$ The $\mathrm{E}_{0}^{\mathrm{U}} \rightarrow \mathrm{E}_{ \pm 1}^{\mathrm{U}}$ relaxation is ultrafast $(<100 \mathrm{fs})$ and is followed by the relaxation of the $\mathrm{E}_{+1}^{\mathrm{U}}$ sublevel, which takes $\sim 700-1000$ fs to reach the $E_{+1}^{\mathrm{L}}$ sublevel when both electron and hole have been excited to higher states. ${ }^{16}$ In such a case the hole is re-excited via Auger energy transfer from excited electrons, and therefore the relaxation takes longer than in the absence of hot electrons (viz., $350 \mathrm{fs}$ ). ${ }^{15}$ The relaxation from the $\mathrm{E}_{ \pm 1}^{\mathrm{L}}$ sublevel to the $\mathrm{E}_{ \pm 2}$ sublevel requires a spin-flip and is therefore relatively slow $\left(0.2-0.4 \mathrm{ps}\right.$ at $300 \mathrm{~K},{ }^{16}$ and tens of picoseconds below $10 \mathrm{~K})^{19}$ As mentioned above, relaxation of the $\mathrm{E}_{ \pm 2}$ sublevel at low temperatures $(<10 \mathrm{~K})$ occurs only radiatively and takes $\sim 0.2$ to $1.4 \mu \mathrm{s}$, depending on the $\mathrm{QD}$ size. $^{30,69}$ 

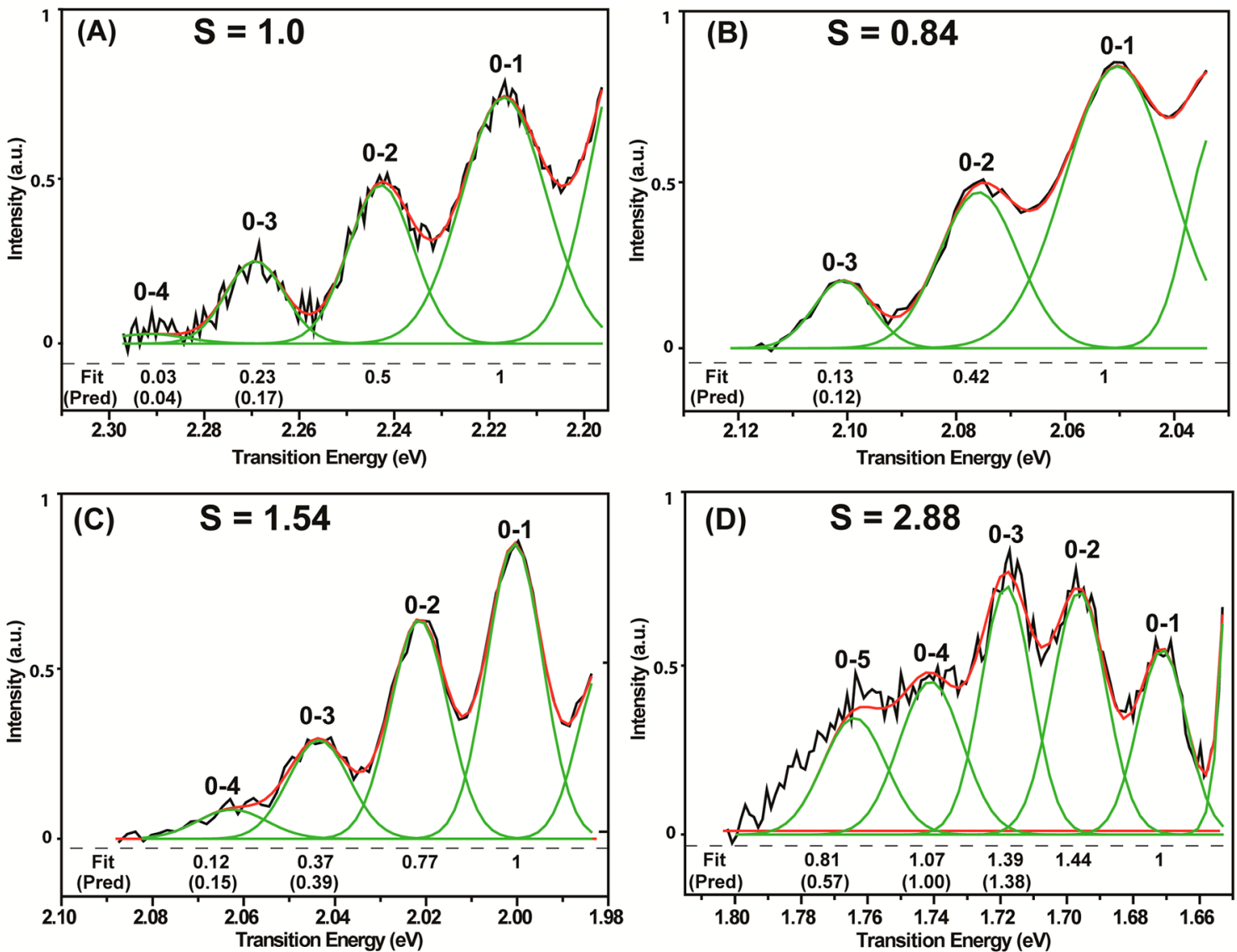

Figure 3. LO-phonon progressions (black lines) observed in the $4.2 \mathrm{~K} \mathrm{PLE} \mathrm{spectra} \mathrm{of} \mathrm{(A)} 3 \mathrm{~nm}$ diameter CdSe NCs $\left(\lambda_{\mathrm{em}}=568 \mathrm{~nm}\right)$, (B) $4.3 \mathrm{~nm}$ diameter CdSe NCs $\left(\lambda_{\mathrm{em}}=614 \mathrm{~nm}\right),(\mathrm{C}) \mathrm{CdTe} / \mathrm{CdSe}$ Type-I ${ }^{1 / 2}$ HNCs $\left(\lambda_{\mathrm{em}}=630 \mathrm{~nm}\right)$, and (D) CdTe $/$ CdSe Type-II HNCs $\left(\lambda_{\mathrm{em}}=756 \mathrm{~nm}\right)$. The LO-phonon progressions were fit to a sum of multiple Gaussians. The LO-phonon replicas are labeled according to the phonon number of the initial and final states $(0-n, n=1,2$, etc.). The estimated Huang-Rhys parameter $S$ is given above the spectra. The numbers under the dashed lines give the relative integrated intensity ratios between the 1-phonon and the $n$-phonon replicas obtained from the fit and predicted on the basis of the estimated $S$ values (Pred).

\subsection{Exciton Transitions and Exciton Relaxation in} Colloidal CdTe/CdSe HNCs. The evolution of the optical properties of $\mathrm{CdTe} / \mathrm{CdSe} \mathrm{HNCs}$ as a function of the CdSe volume fraction has been investigated in detail by our group. ${ }^{57}$ The electron-hole spatial separation in CdTe-CdSe HNCs has been shown to be ultrafast $(0.2-1 \mathrm{ps}) .^{72}$ Hole relaxation in the CdTe part occurs in $\sim 800 \mathrm{ps}$, whereas the electron relaxation in the CdSe part is much faster $(8 \mathrm{ps}) .^{82}$ The hole wave function localizes in the CdTe core already for very small CdSe volume fractions. ${ }^{57}$ Therefore, the evolution of the optical transitions in the HNCs with the growth of the CdSe segment is determined by the localization regime of the electron wave function. For Type- $\mathrm{I}^{1 / 2} \mathrm{CdTe} / \mathrm{CdSe} \mathrm{HNCs}$ the lowest energy electron state is delocalized over the whole HNC (this state is labeled as $1 S_{\mathrm{e}}$ (hetero)). The loss in electron confinement energy results in a shift of all exciton transitions to lower energies. Since the hole levels remain unperturbed and the $1 S_{\mathrm{e}}$ (hetero) is a coherent state delocalized over the whole HNC, the absorption transitions remain direct and therefore distinct and welldefined. $^{57}$ The peaks observed in the PLE spectrum of the Type- $\mathrm{I}^{1 / 2} \mathrm{CdTe} / \mathrm{CdSe}$ HNCs shown in Figure 1 can thus be ascribed to the following absorption transitions: ${ }^{57}$ $1 \mathrm{~S}_{3 / 2(\mathrm{~h})}(\mathrm{CdTe}) \rightarrow 1 \mathrm{~S}_{(\mathrm{e})}($ hetero $)(2.13 \mathrm{eV}) ; 2 \mathrm{~S}_{3 / 2(\mathrm{~h})}(\mathrm{CdTe}) \rightarrow$ $1 \mathrm{~S}_{(\mathrm{e})}($ hetero $)(2.39 \mathrm{eV}) ; 1 \mathrm{P}_{(\mathrm{h})}(\mathrm{CdTe}) \rightarrow 1 \mathrm{P}_{(\mathrm{e})}($ hetero $) \quad(2.7$ $\mathrm{eV})$.
For Type-II CdTe/CdSe HNCs the lowest energy electron state is the $1 S_{(\mathrm{e})}(\mathrm{CdSe})$ [or its equivalent in nanorods, $\left.1 \sum_{(\mathrm{e})}(\mathrm{CdSe})\right] .{ }^{57}$ The lowest energy absorption transition then becomes the spatially indirect transition $1 \mathrm{~S}_{3 / 2(\mathrm{~h})}(\mathrm{CdTe})$ $\rightarrow 1 \mathrm{~S}_{(\mathrm{e})}(\mathrm{CdSe})\left[\right.$ or $1 \mathrm{~S}_{3 / 2(\mathrm{~h})}(\mathrm{CdTe}) \rightarrow 1 \sum_{(\mathrm{e})}(\mathrm{CdSe})$ in branched HNCs]. This results in a further shift of all transitions to lower energies and gives rise to a featureless low-energy absorption tail characteristic of Type-II HNCs. The spatially indirect nature of the exciton in Type-II CdTe/CdSe HNCs also leads to an increase in the Stokes shift values and transition line widths and longer exciton radiative lifetimes. Accordingly, the feature observed beyond $2.25 \mathrm{eV}$ in the PLE spectrum of the Type-II CdTe/CdSe HNCs shown in Figure 1 is assigned to the $1 S_{3 / 2(\mathrm{~h})}(\mathrm{CdTe}) \rightarrow 1 \sum_{(\mathrm{e})}(\mathrm{CdSe})$ absorption transition. ${ }^{57}$

The LO-phonon progression observed in the PLE spectra of the CdTe/CdSe HNCs (Figures 1 and 2) can thus be ascribed to the (partially) spatially indirect exciton transitions $1 S_{3 / 2(\mathrm{~h})}(\mathrm{CdTe}) \rightarrow 1 \mathrm{~S}_{(\mathrm{e})}($ hetero $)$ and $1 \mathrm{~S}_{3 / 2(\mathrm{~h})}(\mathrm{CdTe}) \rightarrow$ $1 \sum_{(\mathrm{e})}(\mathrm{CdSe})$, for Type-I ${ }^{1 / 2}$ and Type-II CdTe/CdSe HNCs, respectively. We note that the lowest energy exciton state in $\mathrm{CdTe} / \mathrm{CdSe} \mathrm{HNCs}\left[1 \mathrm{~S}_{3 / 2(\mathrm{~h})}(\mathrm{CdTe}) 1 \mathrm{~S}_{(\mathrm{e})}\right.$ (hetero) or $\left.1 \mathrm{~S}_{3 / 2(\mathrm{~h})}(\mathrm{CdTe}) \rightarrow 1 \sum_{(\mathrm{e})}(\mathrm{CdSe})\right]$ is 8 -fold degenerate, just like in the CdSe QD case. Exciton fine-structure states are therefore also expected, but studies aiming at their identification are still lacking. It may be expected that the decrease in the electron-hole exchange interaction due to the 
partial spatial separation of the electron and hole wave functions results in less pronounced splitting of the exciton fine-structure sublevels, ${ }^{5,57}$ but this hypothesis remains to be verified. In any case, it is clear that the observed LO-phonon progression is associated with the lowest energy fine-structure states of the $1 \mathrm{~S}_{3 / 2(\mathrm{~h})}(\mathrm{CdTe}) 1 \mathrm{~S}_{(\mathrm{e})}($ hetero $)$ quasi-indirect exciton state of Type-I ${ }^{1 / 2} \mathrm{CdTe} / \mathrm{CdSe} \mathrm{HNCs}$ and the $1 \mathrm{~S}_{3 / 2(\mathrm{~h})}(\mathrm{CdTe})$ $\rightarrow 1 \sum_{(\mathrm{e})}(\mathrm{CdSe})$ spatially indirect exciton state of Type-II CdTe/CdSe HNCs. Similarly to the case of CdSe QDs, the lowest energy line is assigned to a one-LO-phonon replica, rather than to a zero-phonon line.

3.4. Exciton-Phonon Coupling Strength of Colloidal CdSe QDs and CdTe/CdSe HNCs. We now turn to the strength of the ex-ph coupling in the different types of NCs studied in the present work. The magnitude of the ex-ph coupling is typically expressed by using the Huang-Rhys parameter $S$. This parameter is defined under the assumption that the electron-phonon coupling is adiabatic, i.e., the mixing of different electronic states by the phonon coupling is taken to be negligible. ${ }^{83}$ The phonons are then described by an unperturbed Hamiltonian, and the electron-phonon interaction Hamiltonian is taken to be linear in the vibrational amplitude. This leads to a displaced harmonic oscillator model with the dimensionless displacement $\Delta$. This model predicts that at $T=0$ the absorption band shape of an electric dipole transition can be expressed as a series of delta-functions at various energies differing by the phonon frequency $\hbar \omega::^{83}$

$$
I_{a b}(E)=\sum_{n} I_{0} F_{n}(0) \delta\left(E_{0,0}+n \hbar \omega-E\right)
$$

where $E_{0,0}=E_{b, 0}-E_{a, 0}$ gives the energy of the transition between the zero vibrational levels of both initial $(a)$ and final (b) states (i.e., the energy of the zero-phonon line), and $n$ gives the number of phonons generated in the transition (i.e., the $\mathrm{n}^{\text {th }}$ phonon replica of the phonon progression). The term $I_{0}$ corresponds to the intensity of the full band, which is determined by the total $a \rightarrow b$ transition probability $\left(\mid\left\langle\Psi_{b}\left(r_{i}\right.\right.\right.$, $\left.\left.Q_{0}\right)|\mu| \Psi_{a}\left(r_{i}, Q_{0}\right)\right\rangle\left.\right|^{2}$, where $\mu$ is the electric dipole operator). $F_{n}(0)$ is the zero-temperature Franck-Condon factor, which is determined by the square of the vibrational overlap integrals $\left\langle\chi_{(b)}(n) \mid \chi_{a}(0)\right\rangle$ and can be expressed as ${ }^{83}$

$$
F_{n}(0)=\frac{\exp (-S) S^{n}}{n !}
$$

in which $S$ is the Huang-Rhys parameter $\left(S=\Delta^{2} / 2\right)$.

Equations 1 and 2 show that the Huang-Rhys parameter $S$ can be determined from experimental data, provided the intensity ratio between at least two members of the phonon progression is known. Typically, the ratio between the onephonon line and the zero-phonon line $\left(I_{0,1} / I_{0,0}=S\right)$ is used for FLN data, whereas in Raman scattering experiments the ratio between the two-phonon and the one-phonon replicas is used to estimate the magnitude of $S\left(I_{0,2} / I_{0,1}=S / 2\right)$. As discussed above, the zero-phonon line is not observed in the PLE spectra reported here. Therefore, we will use the ratio between the integrated intensities of the two-LO-phonon $(0-2)$ and the one-LO-phonon (0-1) replicas in the PLE spectra to estimate the Huang-Rhys parameter $S$. In order to determine the integrated intensities of the LO-phonon replicas, the contribution of the broad spectral feature was first subtracted from the PLE spectra, by modeling it as a polynomial baseline running from the onset of the PLE spectra (i.e., the lowest excitation energy) to immediately after the highest order observable phonon replica. The intensity of the baseline at the lowest energy limit of the PLE spectra was constrained to the spectral background level (typically just a few counts/s) and increased to the peak intensity immediately after the highest energy observable LO-phonon replica. The uncertainties involved in this process will be discussed in more detail below. The LO-phonon progressions (Figure 3) were subsequently fit to a sum of multiple Gaussian peaks. The $S$ values determined for the $3 \mathrm{~nm}$ diameter CdSe QDs, $4.3 \mathrm{~nm}$ diameter CdSe QDs, Type-I ${ }^{1 / 2} \mathrm{CdTe} / \mathrm{CdSe} H N C s$, and TypeII CdTe/CdSe HNCs are respectively 1.0, 0.84, 1.56, and 2.88 (Figure 3).

The expected ratios between the intensities of the $n$-phonon and the 1-phonon replicas $\left(I_{0, n} / \mathrm{I}_{0,1}\right)$ calculated on the basis of the estimated $S$ parameters are in good agreement with the experimental values determined from the PLE spectra (Figure 3 gives both experimental and predicted values). Some of the predicted LO-phonon replicas were not observed: the 0-4 line for the $4.3 \mathrm{~nm}$ diameter CdSe QDs (predicted intensity: 0.02); the $0-5$ line for the Type- $\mathrm{I}^{1 / 2} \mathrm{CdTe} / \mathrm{CdSe}$ HNCs (predicted: 0.05); and the 0-6 line for the Type-II CdTe/CdSe HNCs (predicted: 0.28 ). The absence of the higher order LO-phonon replicas in the PLE spectra of the $4.3 \mathrm{~nm}$ diameter CdSe QDs and Type- $\mathrm{I}^{1 / 2} \mathrm{CdTe} / \mathrm{CdSe} \mathrm{HNCs}$ can be ascribed to their low intensities. The poor agreement between the predicted and observed intensities for the five-phonon-replica $(0-5)$ in the PLE spectrum of the Type-II CdTe/CdSe HNCs can be attributed to the fact that at sufficiently high energies from the monitored PL energy the spectral selection of subensembles is less effective. Therefore, higher order LO-phonon replicas of larger HNCs spectrally overlap with lower order LO-phonon replicas of smaller HNCs, giving rise to the featureless absorption tail characteristic of spatially indirect exciton transitions.

The good agreement between observed and expected intensity ratios shows that the method used to estimate the Huang-Rhys parameter is robust, and leads to reliable $S$ values. Nevertheless, as mentioned above, the background subtraction involves some uncertainty, which propagates to the intensity ratios of the LO-phonon replicas. To evaluate the impact of this uncertainty on the $S$ values estimated from the PLE spectra, the background was modeled by different baselines, varying from the limit of overestimated $0-1$ intensity (i.e., underestimated $S$ ) to overestimated highest-order LO-phonon replica (i.e., overestimated $S$ ). This analysis is illustrated in the Supporting Information (Figure S3) using the PLE spectrum of the $3 \mathrm{~nm}$ diameter CdSe QDs as a representative example. It is clear that the extreme solutions are physically unrealistic and fail to describe the observed intensities of the LO-phonon progressions. Taking into account the uncertainties involved in the baseline subtraction, the estimated $S$ values can be expressed as $(1.0 \pm 0.2),(0.85 \pm 0.15),(1.5 \pm 0.15)$, and $(2.9 \pm 0.3)$, for the $3 \mathrm{~nm}$ diameter CdSe QDs, $4.3 \mathrm{~nm}$ diameter CdSe QDs, Type-I ${ }^{1 / 2} \mathrm{CdTe} / \mathrm{CdSe}$ HNCs, and Type-II CdTe/CdSe HNCs, respectively. LO-phonon replicas are also observed in the PL spectra (Supporting Information, Figure S2). However, the contribution of the broad band features is more difficult to remove from the PL spectra, since in that case the peak maxima are not clearly observed. For this reason, the quantitative analysis reported here is limited to the LO-phonon progressions observed in the PLE spectra. 
Before comparing the $S$ values determined in the present work to those reported in the literature for CdSe QDs, and discussing the evolution observed in the Huang-Rhys parameter $S$ from CdSe QDs to type-II CdTe/CdSe HNCs, it is useful to first consider the mechanisms behind the electron-phonon coupling in semiconductors and the theoretical predictions concerning the ex-ph coupling in semiconductor NCs. Coupling between electrons (or holes) and phonons can take place through several mechanisms. ${ }^{14,34}$ The volume deformation potential interaction arises from local changes in the crystal's energy bands due to the lattice distortion (i.e., changes in bond lengths and/or angles) created by a phonon. This interaction is more important for coupling to acoustic phonons and in nonpolar materials such as silicon. In polar semiconductors, such as CdSe and CdTe, the dominant coupling mechanism between charge carriers and phonons is the Fröhlich interaction. ${ }^{14,34}$ This interaction arises from the lattice polarization field produced by the relative displacement of positive and negative ions within the unit cell. This induced polarization field is particularly strong for LO phonons. For example, the LO-phonon mode of CdSe has the $\mathrm{Cd}^{2+}$ and $\mathrm{Se}^{2-}$ atoms vibrating out of phase within each unit cell. Since the $\mathrm{Cd}-\mathrm{Se}$ bond is relatively polar, this displacement creates a transient longitudinal dipole moment, which strongly couples to the electric field of the charge carriers. Since the contributions of the electron and hole have opposite signs, the strength of the exciton-LO-phonon coupling reflects the net dipole moment of the exciton, which is determined by the difference in the electron and hole charge distributions (i.e., the spatial distribution of the electron and hole wave functions). ${ }^{34,52}$

To date, the large majority of the theoretical studies aiming at predicting the ex-ph coupling strength of nanoscale excitons in QDs has assumed that the charge distributions of the electron and hole in strongly confining semiconductor nanostructures are almost identical. ${ }^{31-37}$ This assumption implies that the ex-ph coupling should vanish, in striking contrast with experimental studies, which have systematically reported significantly large $S$ values (viz., $0.1-1.0$ ) for a variety of different materials (e.g., $\mathrm{CdS}, \mathrm{PbS}$, InP, InAs, etc.). ${ }^{24,25,32,38-53}$ To reconcile theory and experiment several processes have been invoked: partial separation of the electron and hole wave functions as a result of asymmetric shape, ${ }^{51}$ presence of piezoelectric or strain fields, ${ }^{37,53}$ and the breakdown of the adiabatic approximation. ${ }^{32}$ Further, it has been argued that the coupling is highly sensitive to the exact form of the electron and hole wave functions. ${ }^{52}$ Extrinsic effects such as the presence of charged point defects and surface trapping have also been invoked. $12,13,31,42,43,52$

In the specific case of CdSe QDs, the experimental values reported in the literature for the Huang-Rhys parameter $S$ vary by 2 orders of magnitude (viz., from 0.03 to 3 ). ${ }^{24,25,32,43-50}$ This large disparity can be rationalized by considering that the $S$ values have been deduced from different photophysical properties (viz., homogeneous linewidths, exciton dephasing rates, intensity ratios, etc.), at different temperatures, and by using a wide variety of experimental techniques: spectral hole burning, 4-wave mixing, resonance Raman spectroscopy, FLN, PLE, single NC PL spectroscopy, etc. Different photophysical properties do not necessarily yield comparable $S$ values, since coupling to acoustic phonons and to optical phonons is mediated by different mechanisms. Further, it has been demonstrated by state-resolved femtosecond pump-probe transient absorption spectroscopy that the exciton-phonon coupling strength is strongly state dependent for CdSe QDs, being maximum for the $1 \mathrm{~S}_{(\mathrm{e})} 1 \mathrm{~S}_{3 / 2(\mathrm{~h})}$ exciton state $(S=0.025$ for coupling to LO-phonons and 0.3 for coupling to acoustic phonons) and decreasing for higher-lying states (e.g., for the $1 S_{(\mathrm{e})} 2 S_{1 / 2(\mathrm{~h})} S=0.2$ for coupling to acoustic phonons and nearly zero for coupling to LO-phonons). ${ }^{43}$ Moreover, the $S$ values have also been reported to be strongly temperature dependent, especially for small CdSe QDs (e.g., $S=3.4$ at 1.75 $\mathrm{K}, 1.6$ at $5 \mathrm{~K}$, and 1.1 at $10 \mathrm{~K}$ for $2.3 \mathrm{~nm}$ diameter $\mathrm{CdSe}$ QDs). ${ }^{24}$

Interestingly, PL spectroscopy on single CdSe QDs at $10 \mathrm{~K}$ has shown that the $S$ values vary by a factor $\sim 20$ (from 0.06 to 1.3) within the ensemble, and also fluctuate over time for a single QD. ${ }^{49}$ The fluctuation in the $S$ values was clearly correlated to spectral diffusion, with larger $S$ values being associated with larger spectral jumps to lower energies. The rate and extent of spectral diffusion was shown to be highly power dependent, increasing with the excitation density. For example, PL peak jumps as large as $60 \mathrm{meV}$ were observed at $2.5 \mathrm{KW} /$ $\mathrm{cm}^{2}$ excitation density, whereas at $25 \mathrm{~W} / \mathrm{cm}^{2}$ the spectral jumps were less frequent and much smaller $(0.5 \mathrm{meV}) .{ }^{49}$ Subsequent work demonstrated that similar spectral shifts could be induced by applying external electric fields and that the spectral shift was accompanied by enhanced $S$ values (e.g., from 0.3 to 0.8 for a $60 \mathrm{meV}$ redshift induced by $\left.350 \mathrm{KV} / \mathrm{cm}^{2}\right) .{ }^{84}$ The similarity between the spontaneous spectral diffusion in the absence and presence of an external electric field was interpreted as evidence that spectral diffusion was caused by the transient accumulation of charges in the QDs. ${ }^{84}$ The large $S$ values associated with large spectral redshifts were therefore thought of as being extrinsic in nature. ${ }^{84}$ These results have been recently used to support the idea that $\mathrm{CW}$ frequency-domain spectroscopic techniques (viz., resonance Raman, FLN, PLE, and single dot $\mathrm{PL})$ probe primarily charged or surface trapped states, therefore yielding much larger (extrinsic) $S$ values than those observed by time-domain spectroscopic techniques. ${ }^{12,13,43}$ This proposition is based on the assumption that the lowest energy exciton state in CdSe QDs is always a surface trapped state. We argue that this generalization is inconsistent with a large body of experimental and theoretical data, as discussed above (section 3.2) in relation to the $1 S_{(\mathrm{e})} 1 S_{3 / 2(\mathrm{~h})}$ exciton fine-structure. Moreover, as mentioned above, surface trapped states lead to either PL quenching or strongly red-shifted trap PL, and therefore cannot contribute to PL spectroscopic measurements that make use of spectral selection, such as FLN, PLE, and single dot PL, particularly at the low temperatures $(\leq 10 \mathrm{~K})$ typically used for these experiments. Additionally, QD charging is unlikely to take place under the low excitation densities used in typical PLE and FLN experiments, since single dot PL measurements have clearly established that the spectral diffusion is strongly power dependent and is negligible for low powers. ${ }^{28,49,84}$ We therefore argue that, although the surface trapping hypothesis can adequately rationalize the large $S$ values observed for CdSe QDs by resonance Raman experiments at room temperature, it cannot explain the $S$ values extracted from FLN and PLE measurements at low temperatures. As it will discussed below, the large $S$ values observed in the latter case are intrinsic in nature, and can be understood by considering the $1 S_{(\mathrm{e})} 1 S_{3 / 2(\mathrm{~h})}$ exciton finestructure.

From the discussion above it is evident that the $S$ values determined in the present work can only be meaningfully 
compared to literature values obtained using similar experimental techniques (i.e., PLE and FLN), at low temperatures $(\leq 10 \mathrm{~K})$, and at low excitation densities. The $S$ values of TOPO capped CdSe QDs ranging from 3 to $10 \mathrm{~nm}$ in diameter have been determined by using both PLE and FLN at $10 \mathrm{~K}^{25}$ The $S$ values extracted from FLN spectra were $0.46 \pm 0.11$, whereas PLE spectra yielded $S=0.14 \pm 0.05$. No clear size dependence was observed. ${ }^{25}$ The smaller $S$ values obtained from the PLE spectra can be ascribed to the fact that the lowest energy line was assumed to be the zero-phonon line of the $\mathrm{E}_{ \pm 1}^{\mathrm{L}}$ fine-structure sublevel. ${ }^{25}$ However, as discussed above (section 3.2), this assumption is not justified. If the lowest energy line in the PLE spectra is taken to be the 0-1 LO-phonon replica, the observed $S$ values increase to $\sim 0.3 \pm 0.1$, which are comparable to those extracted from the FLN spectra. The Huang-Rhys parameter of TOPO capped CdSe QDs has also been estimated from FLN spectra in the $1.75-10 \mathrm{~K}$ range, and observed to be temperature- and size-dependent below $10 \mathrm{~K}$, decreasing with increasing temperatures (e.g., $S=3.4$ at $1.75 \mathrm{~K}, 1.6$ at $5 \mathrm{~K}$, and 1.1 at $10 \mathrm{~K}$ for $2.3 \mathrm{~nm}$ diameter CdSe QDs) and sizes (viz., $S$ at $5 K=1.1,1.3$, and 1.6 for $8.3,3.3$, and $2.3 \mathrm{~nm}$ diameter, respectively). ${ }^{24}$ The size dependence is absent at $10 \mathrm{~K}^{24}$ in agreement with subsequent work by Norris and co-workers. ${ }^{25}$ These early observations have been recently corroborated by FLN spectroscopic investigations on $\mathrm{CdSe} / \mathrm{CdS}$ core/shell QDs (core diameter: $3.6 \mathrm{~nm}$; shell thickness: $1 \mathrm{~nm}$ ) in the $1.2-$ $4.2 \mathrm{~K}$ temperature range, under magnetic fields up to $30 \mathrm{~T}^{27}$ The Huang-Rhys parameter is observed to decrease with increasing temperature (viz., $S=1.2$ at $1.2 \mathrm{~K}$ and 0.8 at $4.2 \mathrm{~K}$ ) and with increasing magnetic field (viz., $S=1.2$ at $0 \mathrm{~T}$ and 0.4 at $30 \mathrm{~T}$ ). The exciton lifetimes are also observed to decrease with increasing temperature and magnetic fields, in agreement with previous work by other groups. $26,30,65,68,69,71$

As discussed in section 3.2. above, the temperature- and magnetic field-dependence of the exciton lifetimes of CdSe QDs can be well understood by considering the $1 S_{(\mathrm{e})} 1 S_{3 / 2}(\mathrm{~h})$ exciton fine-structure. ${ }^{26,27,30,65,68,69,71}$ As the temperature decreases, the population of the lower lying $\mathrm{E}_{ \pm 2}$ state ("dark exciton") increases at the expenses of the higher lying $\mathrm{E}_{ \pm 1}^{\mathrm{L}}$ state ("bright exciton"), leading to progressively longer exciton lifetimes, which eventually become equal to the $\mathrm{E}_{ \pm 2}$ radiative lifetime. Similarly, magnetic fields lead to mixing of the $\mathrm{E}_{ \pm 2}$ and $\mathrm{E}_{ \pm 1}^{\mathrm{L}}$ states, and therefore the exciton radiative lifetime decreases with increasing magnetic fields. We note that the dependence of the Huang-Rhys parameter extracted from FLN measurements on both the temperature and the magnetic field strength can easily be explained by assuming that the $\mathrm{E}_{ \pm 2}$ fine-structure state has the strongest ex-ph coupling among the $1 S_{(\mathrm{e})} 1 S_{3 / 2(\mathrm{~h})}$ exciton fine-structure states of wurtzite CdSe QDs. This assumption has been recently verified by low temperature (2$20 \mathrm{~K})$ spectroscopic measurements on single $\mathrm{CdSe} / \mathrm{ZnS}$ core/ shell QDs (core diameter $=8 \mathrm{~nm}$ ), which allowed the direct observation of the lowest two $1 S_{(\mathrm{e})} 1 S_{3 / 2(\mathrm{~h})}$ exciton zero-phonon lines and accompanying LO-phonon replicas. ${ }^{28}$ These experiments clearly demonstrated that the ex-ph coupling of the $\mathrm{E}_{ \pm 2}$ state is much stronger than that of the $\mathrm{E}_{ \pm 1}^{\mathrm{L}}$ state (viz., $S=0.2$ and $\sim 0$, respectively). ${ }^{28}$ The large ex-ph coupling strength for the $\mathrm{E}_{ \pm 2}$ state can be understood by considering that phononassistance is very effective in relaxing selection rules by symmetry breaking/mixing, ${ }^{65,83}$ and will be active even at low temperatures for processes involving the creation of phonons (viz., Stokes LO-phonon replicas, such as those observed in the low temperature FLN and PLE spectra of CdSe QDs). It is therefore clear that the large $S$ values extracted from low temperature $(\leq 10 \mathrm{~K}) \mathrm{FLN}$ and PLE spectra of CdSe QDs reflect the intrinsic ex-ph coupling strength of the $\mathrm{E}_{ \pm 2}$ exciton fine-structure state.

Based on the above discussion it can be concluded that the $S$ values observed in the present work for CdSe QDs at $4.2 \mathrm{~K}$ (viz., $S=1.0 \pm 0.2$ and $0.85 \pm 0.15$, for 3 and $4.3 \mathrm{~nm}$ diameter QDs, respectively) reflect the intrinsic ex-ph coupling, since they are in good agreement with the values reported in the literature for FLN measurements on similarly sized CdSe QDs at comparable temperatures (viz., $S=1.3$ for $3.3 \mathrm{~nm}$ diameter TOPO capped CdSe QDs at $5 \mathrm{~K}^{24}$ and 0.8 for $3.6 \mathrm{~nm}$ diameter $\mathrm{CdSe}$ cores in $\mathrm{CdSe} / \mathrm{CdS}$ core/shell QDs at $4.2 \mathrm{~K}^{27}$ ). The results presented above thus clearly demonstrate that the intrinsic exciton-LO-phonon coupling strength at $4.2 \mathrm{~K}$ increases from CdSe QDs $(S=0.8-1.0)$ to Type- $\mathrm{I}^{1 / 2} \mathrm{CdTe} /$ CdSe HNCs $(S=1.5)$ and from these to Type-II CdTe/CdSe HNCs $(S=2.9)$. The essential difference between these three types of colloidal NCs is that the overlap between the electron and hole wave functions progressively decreases from single composition CdSe QDs to Type-II CdTe/CdSe HNCs (figure 4).

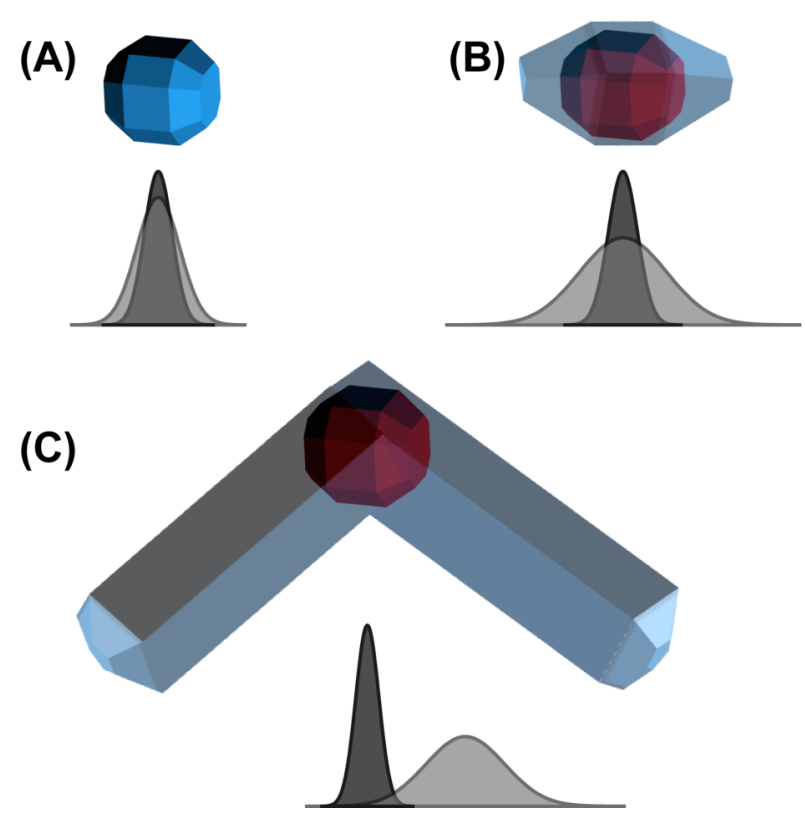

Figure 4. Schematic illustration of the spatial distribution of the electron and hole wave functions (light and dark gray, respectively) in the NCs investigated in this work. (A) CdSe QDs, (B) CdTe/CdSe Type-I ${ }^{1 / 2}$ HNCs, and (C) CdTe/CdSe Type-II HNCs.

The CdSe QDs investigated here have radii of 1.5 and 2.15 $\mathrm{nm}$ and are thus in the strong quantum confinement regime, since the exciton Bohr radius in bulk CdSe is $4.9 \mathrm{~nm} .{ }^{60}$ We note, however, that these sizes are not sufficiently small to induce significant confinement effects in the hole wave function (electron and hole Bohr radii in bulk CdSe are 3.8 and $1.1 \mathrm{~nm}$, respectively). ${ }^{60}$ This implies that the distribution of the hole and the electron wave functions in CdSe QDs may be considerably different for different exciton (fine-structure) states. The hole wave function in $\mathrm{CdTe} / \mathrm{CdSe} \mathrm{HNCs}$ is confined to the CdTe core, already for very small $\mathrm{CdSe}$ volume fractions. ${ }^{57}$ Therefore, the evolution of the optical properties of $\mathrm{CdTe} / \mathrm{CdSe} \mathrm{HNCs}$ with increasing CdSe volume fraction is 
determined by the localization regime of the electron wave function. ${ }^{57}$ In Type- $\mathrm{I}^{1 / 2} \mathrm{CdTe} / \mathrm{CdSe}$ HNCs the electron wave function is delocalized over the whole HNC, whereas in TypeII CdTe/CdSe HNCs the electron wave function is centered in the CdSe segment of the HNC. ${ }^{57}$ As a result of the change in the spatial distribution of the electron and hole wave functions, the electron-hole overlap in $\mathrm{CdTe} / \mathrm{CdSe} \mathrm{HNCs}$ progressively decreases with the growth of the CdSe segment. This leads to an increasingly larger exciton polarization, which in turn enhances the ex-ph coupling strength. The present results provide novel insights into the nature of spatially indirect exciton transitions, showing that the featureless absorption tail typically associated with the formation of spatially indirect excitons consists primarily of phonon-assisted exciton transitions, in agreement with the model proposed in ref 57.

\section{CONCLUSIONS}

In conclusion, LO-phonon progressions are clearly observed in the low temperature $(4.2 \mathrm{~K})$ PLE spectra of different types of colloidal NCs. The strength of the exciton-LO-phonon coupling, as reflected by the Huang-Rhys parameter $S$, is shown to increase from CdSe QDs $(S=0.8-1.0)$ to Type- $I^{1 / 2}$ CdTe/CdSe HNCs $(S=1.5)$, and from these to Type-II $\mathrm{CdTe} / \mathrm{CdSe}$ HNCs $(S=2.9)$. This trend can be explained by the decrease in the electron-hole wave function overlap that accompanies the gradual increase in the CdSe volume fraction in the HNCs, as the electron wave function gradually localizes in the CdSe segment. The decrease in the electron-hole overlap leads to larger exciton polarizations, which in turn enhance the exciton-LO-phonon coupling via the Fröhlich interaction. The results provide novel insights in the nature of spatially indirect exciton transitions, showing that the featureless absorption tail typically associated with the formation of spatially indirect excitons consists primarily of phonon-assisted exciton transitions. Moreover, this work demonstrates that compositional control of semiconductor heteronanocrystals can be used as a very effective tool to tailor the exciton-phonon coupling strength of nanoscale excitons.

\section{ASSOCIATED CONTENT}

\section{S Supporting Information}

Experimental details regarding the synthesis of the $3 \mathrm{~nm} \mathrm{CdSe}$ QDs, main characteristics of the colloidal CdTe/CdSe HNCs investigated here, and additional figures. This material is available free of charge via the Internet at http://pubs.acs.org.

\section{AUTHOR INFORMATION}

\section{Corresponding Author}

*E-mail: c.demello-donega@uu.nl.

\section{Notes}

The authors declare no competing financial interest.

\section{REFERENCES}

(1) Donega, C. d. M. Chem. Soc. Rev. 2011, 40, 1512-1546.

(2) Pandey, A.; Guyot-Sionnest, P. Science 2008, 322, 929-932.

(3) Lo, S. S.; Mirkovic, T.; Chuang, C.; Burda, C.; Scholes, G. D. Adv. Mater. 2011, 23, 180-197.

(4) Oron, D.; Kazes, M.; Banin, U. Phys. Rev. B. 2007, 75, 035330.

(5) Brovelli, S.; Schaller, R. D.; Crooker, S. A.; Garcia-Santamaria, F.; Chen, Y.; Viswanatha, R.; Hollingsworth, J. A.; Htoon, H.; Klimov, V. I. Nat. Comm. 2011, 2, 280.
(6) Groeneveld, E.; van Berkum, S.; van Schooneveld, M. M.; Gloter, A.; Meeldijk, J. D.; van den Heuvel, D. J.; Gerritsen, H. C.; de Mello Donega, C. Nano Lett. 2012, 12, 749-757.

(7) Tyagi, P.; Kambhampati, P. J. Phys. Chem. C 2012, 116, 81548160.

(8) Reiss, P.; Protière, M.; Li, L. Small 2009, 5, 154-168.

(9) Talapin, D. V.; Lee, J.; Kovalenko, M. V.; Shevchenko, E. V. Chem. Rev. 2010, 110, 389-458.

(10) Klimov, V. I. Annu. Rev. Phys. Chem. 2007, 58, 635-673.

(11) Costi, R.; Saunders, A. E.; Banin, U. Angew. Chem., Int. Ed. 2010, 49, 4878-4897.

(12) Kambhampati, P. J. Phys. Chem. C 2011, 115, 22089-22109.

(13) Kambhampati, P. Acc. Chem. Res. 2012, 44, 1-13.

(14) Stroscio, M. A.; Dutta, M. Phonons in Nanostructures; Cambridge University Press: New York, 2001.

(15) Hendry, E.; Koeberg, M.; Wang, F.; Zhang, H.; de Mello Donegá, C.; Vanmaekelbergh, D.; Bonn, M. Phys. Rev. Lett. 2006, 96, 057408 .

(16) Wang, H.; de Mello Donegá, C.; Meijerink, A.; Glasbeek, M. J. Phys. Chem. B 2006, 110, 733-737.

(17) Beard, M. C.; Midgett, A. G.; Hanna, M. C.; Luther, J. M.; Hughes, B. K.; Nozik, A. J. Nano Lett. 2010, 10, 3019-3027.

(18) Boulesbaa, A.; Issac, A.; Stockwell, D.; Huang, Z.; Huang, J.; Guo, J.; Lian, T. J. Am. Chem. Soc. 2007, 129, 15132-15133.

(19) Hannah, D. C.; Dunn, N. J.; Ithurria, S.; Talapin, D. V.; Chen, L. X.; Pelton, M.; Schatz, G. C.; Schaller, R. D. Phys. Rev. Lett. 2011, 107, 177403.

(20) Houtepen, A. J.; Kockmann, D.; Vanmaekelbergh, D. Nano Lett. 2008, 8, 3516-3520.

(21) Rogach, A. L.; Klar, T. A.; Lupton, J. M.; Meijerink, A.; Feldmann, J. J. Mater. Chem. 2009, 19, 1208-1221.

(22) Wuister, S. F.; Koole, R.; de Mello Donegá, C.; Meijerink, A. J. Phys. Chem. B 2005, 109, 5504-5508.

(23) Liptay, T. J.; Marshall, L. F.; Rao, P. S.; Ram, R. J.; Bawendi, M. G. Phys. Rev. B 2007, 76, 155314.

(24) Nirmal, M.; Murray, C. B.; Bawendi, M. G. Phys. Rev. B. 1994 50, 2293-2300.

(25) Norris, D. J.; Efros, A. L.; Rosen, M.; Bawendi, M. G. Phys. Rev. B 1996, 53, 16347-16354.

(26) Nirmal, M.; Norris, D. J.; Kuno, M.; Bawendi, M. G.; Efros, Al. L.; Rosen, M. Phys. Rev. Lett. 1995, 75, 3728-3731.

(27) Wijnen, F. J. P.; Blokland, J. H.; Chin, P. T. K.; Christianen, P. C. M.; Maan, J. C. Phys. Rev. B 2008, 78, 235318.

(28) Biadala, L.; Louyer, Y.; Tamarat, Ph.; Lounis, B. Phys. Rev. Lett. 2009, 103, 037404.

(29) Huxter, V. M.; Scholes, G. D. J. Chem. Phys. 2010, 132, 104506.

(30) Oron, D.; Aharoni, A.; de Mello Donegá, C.; van Rijssel, J.; Meijerink, A.; Banin, U. Phys. Rev. Lett. 2009, 102, 177402.

(31) Nomura, S.; Kobayashi, T. Phys. Rev. B 1992, 45, 1305-1316.

(32) Fomin, V. M.; Gladilin, V. N.; Devreese, J. T.; Pokatilov, E. P.; Balaban, S. N.; Klimin, S. N. Phys. Rev. B 1998, 57, 2415-2425.

(33) Melnikov, D. V.; Fowler, W. B. Phys. Rev. B 2001, 64, 245320.

(34) Delerue, C.; Lannoo, M. Nanostructures: Theory and Modelling; Springer-Verlag: Berlin, 2004.

(35) Kelley, A. M. ACS Nano 2011, 5, 5254-5262.

(36) Schmitt-Rink, S.; Miller, D. A. B.; Chemla, D. S. Phys. Rev. B 1987, 35, 8113-8125.

(37) Heitz, R.; Mukhametzhanov, I.; Stier, O.; Madhukar, A.; Bimberg, D. Phys. Rev. Lett. 1999, 83, 4654-4657.

(38) Martin, J.; Cichos, F.; Huisken, F.; von Borczyskowski, C. Nano Lett. 2008, 8, 656-660.

(39) Kwok, S. H.; Yu, P. Y.; Tung, C. H.; Zhang, Y. H.; Li, M. F.; Peng, C. S.; Zhou, J. M. Phys. Rev. B 1999, 59, 4980-4984.

(40) Shiang, J. J.; Risbud, S. H.; Alivisatos, A. P. J. Chem. Phys. 1993, $98,8432-8442$.

(41) Seong, M. J.; Micic, O. I.; Nozik, A. J.; Mascarenhas, A.; Cheong, H. M. Appl. Phys. Lett. 2003, 82, 185-187.

(42) Krauss, T. D.; Wise, F. W. Phys. Rev. B 1997, 55, 9860-9865. 
(43) Sagar, D. M.; Cooney, R. R.; Sewall, S. L.; Dias, E. A.; Barsan, M. M.; Butler, I. S.; Kambhampati, P. Phys. Rev. B 2008, 77, 235321.

(44) Lange, H.; Mohr, M.; Artemyev, M.; Woggon, U.; Niermann, T.; Thomsen, C. Phys. Status Solidi B 2010, 247, 2488-2497.

(45) Salvador, M. R.; Hines, M. A.; Scholes, G. D. J. Chem. Phys. 2003, 118, 9380-9388.

(46) Klein, M. C.; Hache, F.; Ricard, D.; Flytzanis, C. Phys. Rev. B 1990, 42, 11123-11132.

(47) Alivisatos, A. P.; Harris, T. D.; Carroll, P. J.; Steigerwald, M. L.; Brus, L. E. J. Chem. Phys. 1989, 90, 3463-3468.

(48) Sun, Z.; Swart, I.; Delerue, C.; Vanmaekelbergh, D.; Liljeroth, P. Phys. Rev. Lett. 2009, 102, 196401.

(49) Empedocles, S. A.; Norris, D. J.; Bawendi, M. G. Phys. Rev. Lett. 1996, 77, 3873-3876.

(50) Fernée, M. J.; Littleton, B. N.; Cooper, S.; Rubinsztein-Dunlop, H.; Gómez, D. E.; Mulvaney, P. J. Phys Chem. C 2008, 112, 18781884.

(51) Garcia-Cristobal, A.; Minnaert, A. W. E.; Fomin, V. M.; Devreese, J. T.; Silov, A. Yu.; Haverkort, J. E. M.; Wolter, J. H. Phys. Status Solidi B 1999, 215, 331-336.

(52) Lemaitre, A.; Ashmore, A. D.; Finley, J. J.; Mowbray, D. J.; Skolnick, M. S.; Hopkinson, M.; Krauss, T. F. Phys. Rev. B 2001, 63, 161309(R).

(53) Kalliakos, S.; Zhang, X. B.; Taliercio, T.; Lefebvre, P.; Gil, B.; Grandjen, N.; Damilano, B.; Massies, J. Appl. Phys. Lett. 2002, 80, 428-430.

(54) Zhang, Q.; Zhang, J.; Utama, M. I. B.; Peng, B.; De la Mata, M.; Arbiol, J.; Xiong, Q. Phys. Rev. B 2012, 85, 085418.

(55) Chilla, G.; Kipp, T.; Menke, T.; Heitmann, D.; Nikolic, M.; Fromsdorf, A.; Kornowski, A.; Förster, S.; Weller, H. Phys. Rev. Lett. 2008, 100, 057403.

(56) Chin, P. T. K.; de Mello Donegá, C.; van Bavel, S. S.; Meskers, S. C. J.; Sommerdijk, N. A. J. M.; Janssen, R. A. J. J. Am. Chem. Soc. 2007, 129, 14880-14886.

(57) Donega, C. d. M. Phys. Rev. B 2010, 81, 165303.

(58) Donega, C. d. M.; Hickey, S. G.; Wuister, S. F.; Vanmaekelbergh, D.; Meijerink, A. J. Phys. Chem. B 2003, 107, 489496.

(59) Qu, L.; Peng, X. J. Am. Chem. Soc. 2002, 124, 2049-2055.

(60) Donega, C. d. M.; Koole, R. J. Phys. Chem. C 2009, 113, 65116520.

(61) Donega, C. d. M.; Meijerink, A.; Blasse, G. J. Lumin. 1994, 62, 189-201.

(62) Rössler, U., Ed.; II-VI and I-VII Compounds; Semimagnetic compounds. Landolt-Börnstein - Group III Condensed Matter; Springer-Verlag: Berlin, 1999; Vol. 41.

(63) Horodysky, P.; Hlidek, P. Phys. Status Solidi B 2006, 243, 494501.

(64) Norris, D. J.; Bawendi, M. G. Phys. Rev. B 1996, 53, 1633816346.

(65) Efros, A. L.; Rosen, M.; Kuno, M.; Nirmal, M.; Norris, D. J.; Bawendi, M. G. Phys. Rev. B 1996, 54, 4843-4856.

(66) Franceschetti, A.; Fu, H.; Wang, L. W.; Zunger, A. Phys. Rev. B 1999, 60, 1819-1829.

(67) Leung, K.; Pokrant, S.; Whaley, K. B. Phys. Rev. B 1998, 57, 12291-12301.

(68) Labeau, O.; Tamarat, P.; Lounis, B. Phys. Rev. Lett. 2003, 90, 257404.

(69) Donega, C. d. M.; Bode, M.; Meijerink, A. Phys. Rev. B 2006, 74, 085320 .

(70) Norris, D. J.; Bawendi, M. G. J. Chem. Phys. 1995, 103, 52605268 .

(71) Moreels, I.; Raino, G.; Gomes, R.; Hens, Z.; Stoferle, T.; Mahrt, R. F. ACS Nano 2011, 5, 8033-8039.

(72) He, J.; Lo, S. S.; Kim, J.; Scholes, G. D. Nano Lett. 2008, 8, 4007-4013.

(73) Kuno, M.; Lee, J. K.; Dabbousi, B. O.; Mikulec, F. V.; Bawendi, M. G. J. Chem. Phys. 1997, 106, 9869-9882.
(74) Jones, M.; Lo, S. S.; Scholes, G. D. J. Phys. Chem. C 2009, 113, $18632-18642$.

(75) Valerini, D.; Creti, A.; Lomascolo, M.; Manna, L.; Cingolani, R.; Anni, M. Phys. Rev. B 2005, 71, 235409.

(76) Pandey, A.; Guyot-Sionnest, P. Science 2008, 322, 929-932.

(77) Koole, R.; Schapotschnikow, P.; de Mello Donegá, C.; Vlugt, T. J. H.; Meijerink, A. ACS Nano 2008, 2, 1703-1714.

(78) Matylitsky, V. V.; Dworak, L.; Breus, V. V.; Basché, T.; Wachtveitl, J. J. Am. Chem. Soc. 2009, 131, 2424-2424.

(79) Wuister, S. F.; Swart, I.; van Driel, F.; Hickey, S. G.; de Mello Donegá, C. Nano Lett. 2003, 3, 503-507.

(80) Crooker, S. A.; Barrick, T.; Hollingsworth, J. A.; Klimov, V. I. Appl. Phys. Lett. 2003, 82, 2793-2795.

(81) Cooney, R. R.; Sewall, S. L.; Dias, E. A.; Sagar, D. M.; Anderson, K. E. H.; Kambhampati, P. Phys. Rev. B 2007, 75, 245311.

(82) Chuang, C. H.; Doane, T. L.; Lo, S. S.; Scholes, G. D.; Burda, C. ACS Nano 2011, 5, 6016-6024.

(83) Henderson, B.; Imbusch, G. F. Optical Spectroscopy of Inorganic Solids; Oxford University Press: Oxford, U.K., 1989.

(84) Empedocles, S. A.; Bawendi, M. G. Science 1997, 278, 21142117. 